\title{
Non-Abelian Charged Nodal Links in a Dielectric Photonic Crystal
}

\author{
Haedong Park, Stephan Wong, Xiao Zhang, and Sang Soon Oh*
}

Cite This: ACS Photonics 2021, 8, 2746-2754

Read Online

ABSTRACT: A nodal link is a special form of a line degeneracy (a nodal line) between adjacent bands in the momentum space of a three-dimensional topological crystal. Unlike nodal chains or knots, a nodal link consists of two or more mutually linked rings that do not touch each other. Recent studies on non-Abelian band topology revealed that the topological charges of the nodal links can have the properties of quaternions. However, a photonic crystal that has a nodal link with non-Abelian charges has not been reported. Here, we propose dielectric photonic crystals in the form of double diamond structures that realize the nodal links in the momentum space. By examining the evolution of the eigenstate correlations along closed loops that enclose the nodal line(s) of the

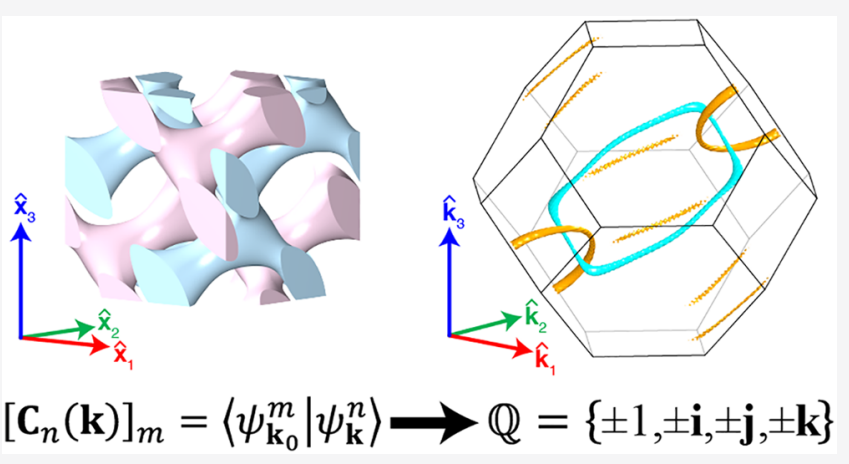
links, their non-Abelian topological charges are also analyzed. The proposed design scheme and theoretical approach in this work will allow for experimental observation of photonic non-Abelian charges in purely dielectric materials and facilitate the control of the degeneracy in complex photonic structures.

KEYWORDS: photonic crystal, topological physics, nodal link, non-Abelian, quaternions, correlations

$\mathrm{T}$ he study of topological crystals is concerned with degenerate states in the band structures. In many cases, the dimensions of the degeneracies are zero-dimensional (OD) such as Dirac ${ }^{1-4}$ or Weyl points ${ }^{5-13}$ or one-dimensional (1D) such as nodal lines. ${ }^{14-20}$ Such topological crystals can induce interesting boundary states, such as one-way edge/surface states, $^{21-23}$ surface localizations, ${ }^{24-26}$ arc surface states, ${ }^{27-30}$ drumhead surface states,,$^{31-35}$ and bound states in the continuum (BIC). ${ }^{36}$ The nodal lines, protected by $\mathcal{P} \mathcal{T}$ symmetry which is the product of time-reversal symmetry ( $\mathcal{T})$ and parity $(\mathcal{P})$ inversion, ${ }^{37}$ are categorized as nodal rings, ${ }^{31,32,38}$ nodal chains, ${ }^{33,34,39-41}$ nodal links, ${ }^{33-35,41-45}$ and nodal knots. ${ }^{35,45,46}$ A nodal ring is a simple closed-loop form of the nodal line. Two or more nodal rings form a nodal chain when they intersect at a single point..$^{33,34,39-41}$ If two or more nodal rings are connected without any touch, they form a nodal link, for example, the Hopf link. ${ }^{33-35,41-45}$ A nodal knot is a single closed loop that cannot be deformed into the unknot state by Reidemeister moves, for example, the trefoil knot. $^{35,45,46}$ Extensive efforts have been recently made to find nodal lines in topological metals, ${ }^{16,39,44}$ semimetals, $^{15,33,41,43,45,46}$ phononic crystals, ${ }^{31}$ electrical circuits, ${ }^{35}$ and network devices. ${ }^{47}$ Several studies have discussed topological invariants of the nodal lines such as the non-Abelian BerryWilczek-Zee (BWZ) connection, ${ }^{48}$ winding number, ${ }^{45,49}$ and Chern number. ${ }^{49}$ Very recently, a study on non-Abelian band topology showed that the topological charges of the nodal lines, including the nodal links or nodal chains, can be described as quaternions. ${ }^{16}$

Despite the abundance of studies on the nodal lines ${ }^{13,17,32,40,42,50}$ and the non-Abelian band topology in photonics, ${ }^{42}$ there has not been any textbook example on the nodal link. A photonic crystal can be such an example if (i) its output nodal lines completely satisfy the aforementioned definition of the nodal link ${ }^{45}$ and (ii) the nodal links of the photonic crystal have non-Abelian topological charges. ${ }^{16}$ The existing photonic crystals on the nodal lines do not completely fulfill at least one of these. In particular, although the second condition has been tested with using the effective $3 \times 3$ Hamiltonian derived from Maxwell's equations, ${ }^{42}$ there is not any precedent in which the non-Abelian topological charges are calculated based on the full-vector field form of eigenstates computed for a photonic crystal. Additionally, most of the existing photonic studies on the nodal line, ${ }^{17}$ nodal ring, ${ }^{32,38}$ nodal chain, ${ }^{40,50}$ or nodal links ${ }^{42}$ are commonly limited to the metallic structures with mirror symmetry.

In this work, we theoretically demonstrate the nodal link with non-Abelian charges by using a dielectric photonic crystal. First, we introduce our double diamond structure, which has inversion symmetries but does not have mirror symmetry. Then, the nodal link in the three-dimensional (3D)

Received: June 14, 2021

Published: August 30, 2021

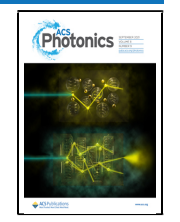


(a)
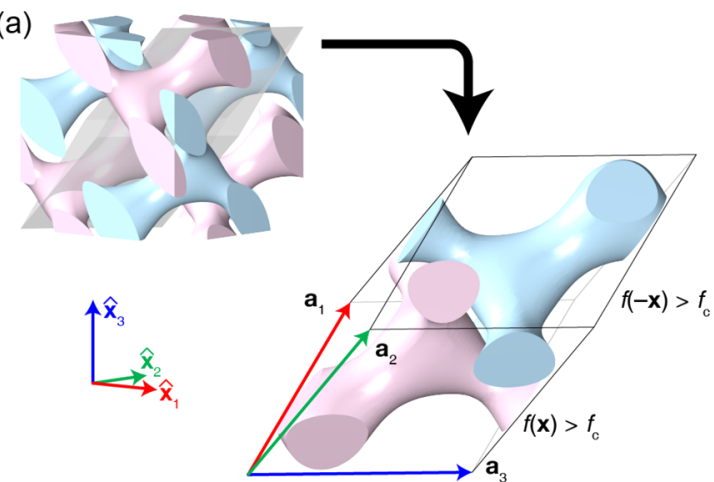

(b)

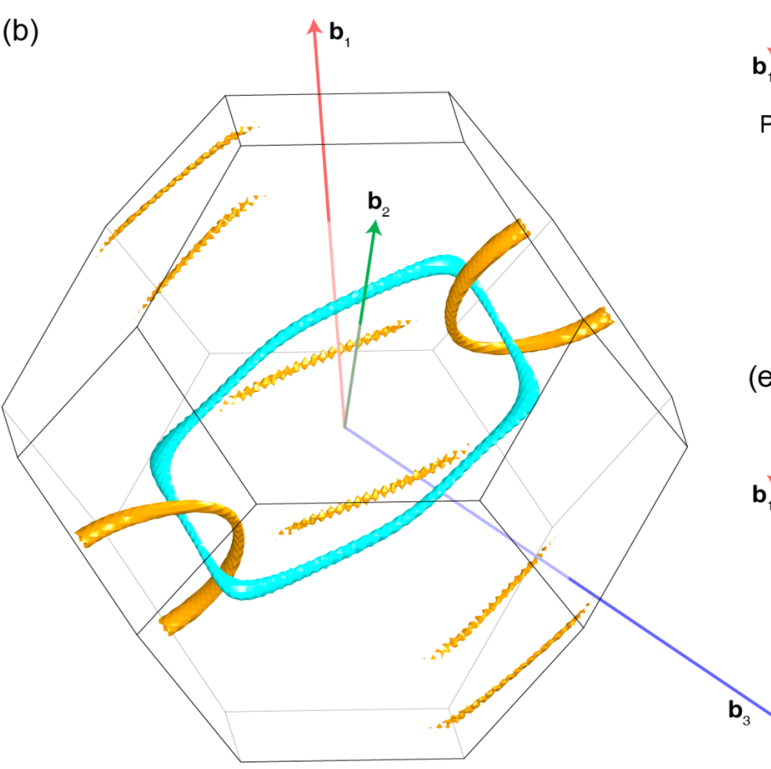

(c)

(d)
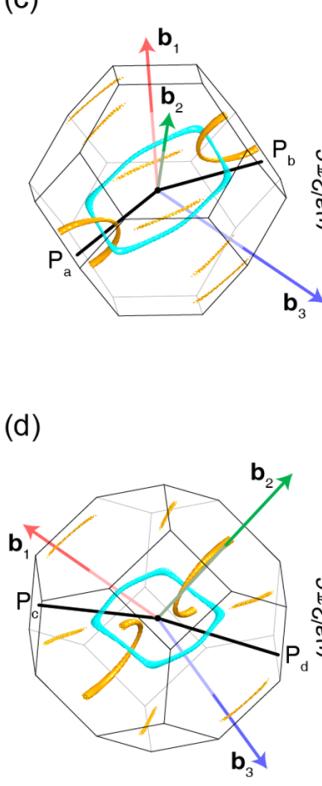

(e)

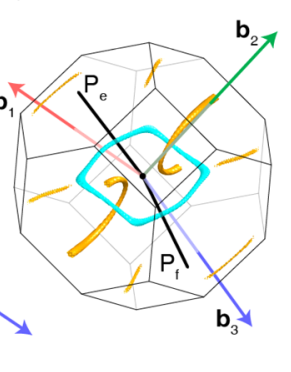

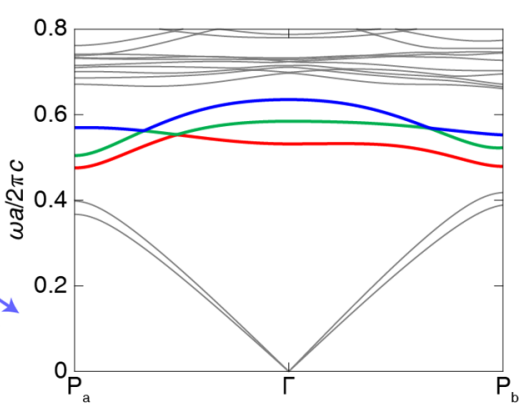
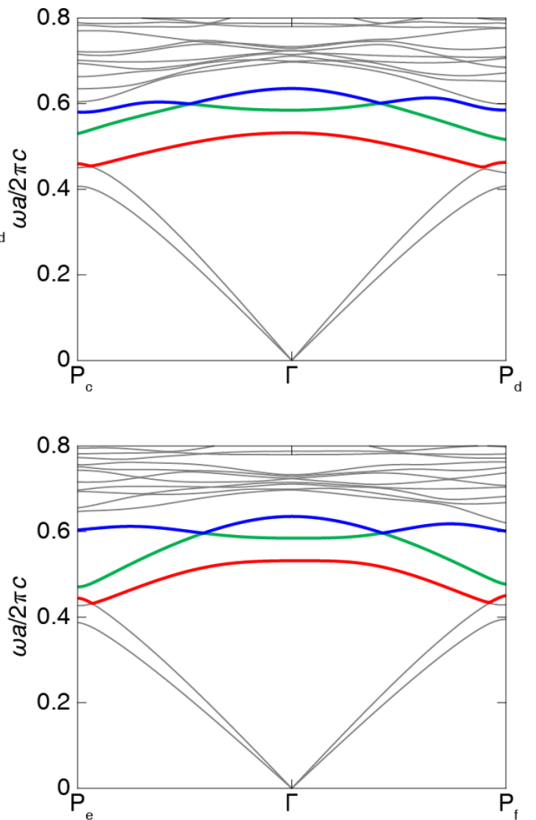

Figure 1. Double diamond photonic crystal and its nodal link. (a) Real space geometry with $\mathbf{A}=[1.19,1.37,1.28], \gamma=0.08$, and $f_{c}=1.85$. Dielectric permittivity is 16.0 for both structures. The FCC primitive cell is defined by $\mathbf{a}_{1}=a / 2[0,1,1], \mathbf{a}_{2}=a / 2[1,0,1]$, and $\mathbf{a}_{3}=a / 2[1,1,0]$. (b) Nodal link in 3D momentum space. The orange and cyan nodal rings are respectively formed by the third and fourth bands and fourth and fifth bands in the band structure. (c-e) Band structures (right) along the several paths (left).

momentum space of this structure is discussed. The nonAbelian topological features of the nodal link are characterized by considering a loop that encloses one or more sections of the link and examining the correlations of the eigenstates.

\section{RESULTS AND DISCUSSION}

Nodal Link. The realization of the nodal link in a dielectric photonic crystal starts with taking the well-known diamond structure $^{51}$ and subsequently breaking its several geometrical symmetries as will be explained below. For the face-centered cubic (FCC) primitive cell whose lattice vectors are $\mathbf{a}_{1}=a /$ $2[0,1,1], \mathbf{a}_{2}=a / 2[1,0,1]$, and $\mathbf{a}_{3}=a / 2[1,1,0]$, our bicontinuous photonic crystal is defined by a set of $\mathbf{x}=\left[x_{1}, x_{2}\right.$, $\left.x_{3}\right]$ such that $f(\mathbf{x})>f_{c}>0$ or $f(-\mathbf{x})>f_{c}>0$ where $f(\mathbf{x})$, the triply periodic level surface, is expressed as

$$
f(\mathbf{x})=\sin \left(X_{1}+X_{2}+X_{3}\right)+\sum_{i=1}^{3} A_{i} \sin \left(X_{1}+X_{2}+X_{3}-2 X_{i}\right)
$$

Here, $\mathbf{X}=\left[X_{1}, X_{2}, X_{3}\right]=(2 \pi / a)(\mathbf{x}-\gamma \mathbf{a} / 2)$ is a normalized local coordinate defined with the lattice constant $a$, global coordinates $\mathbf{x}$, the summation of the lattice vectors $\mathbf{a}=\sum_{i=1}^{3} \mathbf{a}_{i}$, and the coefficient $\gamma$ that describes the translation of one diamond from the other along the $[1,1,1]$-direction. By the inequalities mentioned above, this photonic crystal consists of two inversion symmetric single diamonds, and they do not intersect each other like other bicontinuous structures. ${ }^{5,12,13,52-55}$ If $A_{1}=A_{2}=A_{3}=1$, the space group of each part becomes $F d \overline{3} m$ (No. 227), and each diamond is identical to the well-known diamond structure. ${ }^{51}$ If $\gamma=0$ is also satisfied, the space group of the total structure becomes $P_{n} \overline{3} m$ (No. 224) whose lattice constant is only $a / 2 .{ }^{52-55}$ Under this condition, a perfect nodal link is not observed because of extra degeneracies (see section 1.1, Supporting Information). To remove the redundant degeneracies, several geometrical symmetries should be broken by the following two modifications. Setting the coefficients $A_{i}$ different from each other and not equal to 1 destroys all the symmetries except translational and inversion symmetries and makes the structure anisotropic along all directions. Under this symmetry breaking, the two single diamonds are still mutual-inversion symmetric, and each diamond is also self-inversion symmetric that the inverse of a single diamond coincides itself. Here, the selfinversion symmetric points of the two single diamonds are identical. The second modification is setting nonzero $\gamma$, which splits the self-inversion symmetric points along the $[1,1,1]$ direction. This also eliminates the translational symmetry with 
(a)

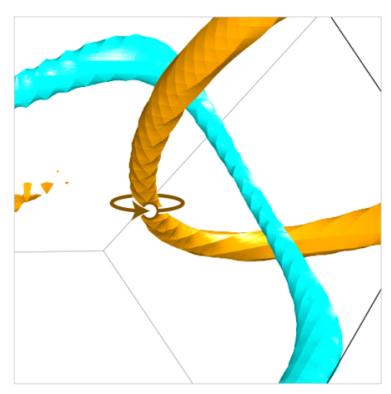

(b)
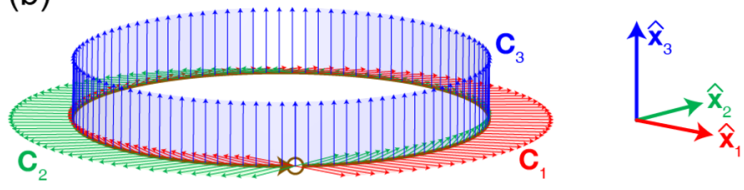

(d)
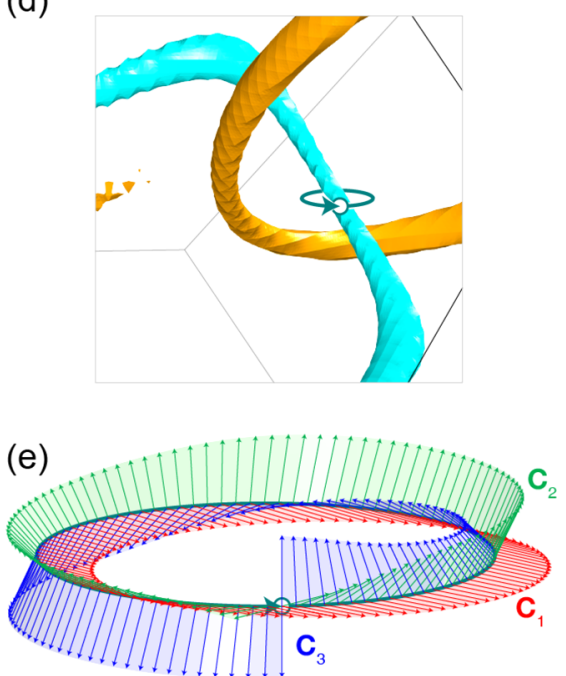

(f)

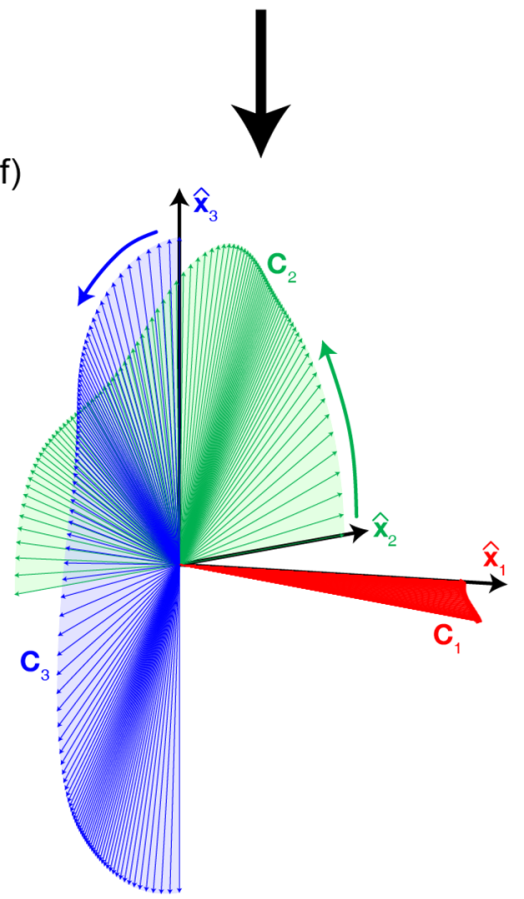

Figure 2. Derivation of the topological charges $\mathbf{k}$ and i. (a, d) Closed loops which enclose the orange and cyan nodal rings, respectively. Their winding directions and starting points $\mathbf{k}_{0}$ are also marked as arrows and circles, respectively. (b, e) Correlations $\mathbf{C}_{n}$ from the eigenstates, along the closed loops. Only their real parts are plotted. $(c, f)$ Correlations $\mathbf{C}_{n}$ whose vector tails are collected at the origin to see their topological charges. In $(b, c)$ and $(e, f)$, we set the arbitrary orthonormal directions as $\hat{\mathbf{x}}_{1}, \hat{\mathbf{x}}_{2}$, and $\hat{\mathbf{x}}_{3}$.

the unit vector $\langle a / 2,0,0\rangle$ so that the primitive cubic cell with $(a / 2)^{3}$ doesn't have to be used, but the above FCC cell has to be used (the symmetries of the double diamond structures are discussed in section 1.2, Supporting Information). The mutualinversion symmetry by two single diamond structures survives, which is a necessary condition for the formation of a nodal link. ${ }^{13,37}$ Each diamond structure in these configurations is depicted in Figure 1a; the sets of $\mathbf{x}$ that satisfy $f(\mathbf{x})>f_{c}$ and $f(-\mathbf{x})>f_{c}$ are given as the pink and sky-blue colored structures, respectively.

The 3D photonic band structure for the double diamond photonic crystal has multiple degeneracies that form a link. To probe all the degeneracies, we calculated the photonic band structure by using the MIT Photonic-Bands (MPB) package. ${ }^{56}$ We regarded any point as degenerated by the adjacent two bands if the normalized frequency difference $(\Delta \omega a / 2 \pi c)$ of these bands at the point is smaller than a critical tolerance, 0.0045 . Sets of the degeneracies formed by the third and fourth bands and fourth and fifth bands appear as rings, depicted as orange and cyan shapes, respectively, in Figure 1b. (Frequency difference data of the nodal link are in section 8, Supporting Information.) These two rings are mutually linked like a ship chain. Features of this result are as follows: (i) This link is infinitely repeated along the connection direction because of the periodicity of the first Brillouin zone. The cyan ring centered at the $\Gamma$-point connects the orange rings spanning the zone boundary. (ii) The link is formed not along, but avoiding, the high symmetry lines. Each band structure that shows degeneracies in Figure $1 c-e$ is the result obtained along the path intersecting the nodal link and connecting two points on 
(a)

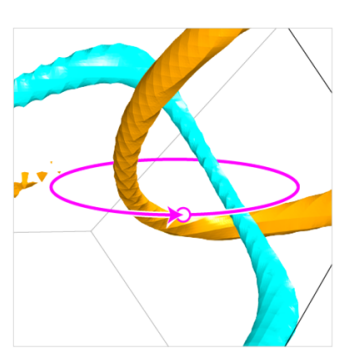

(b)

(c)
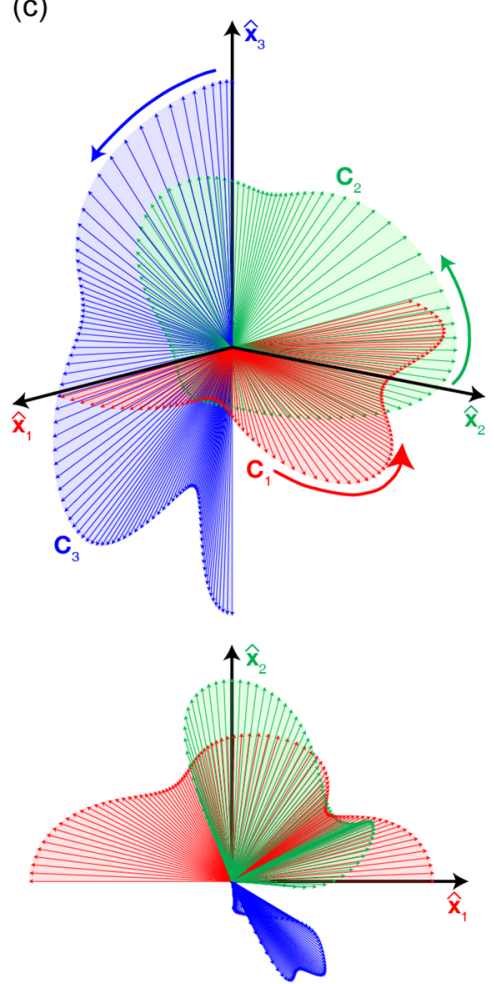

(d)
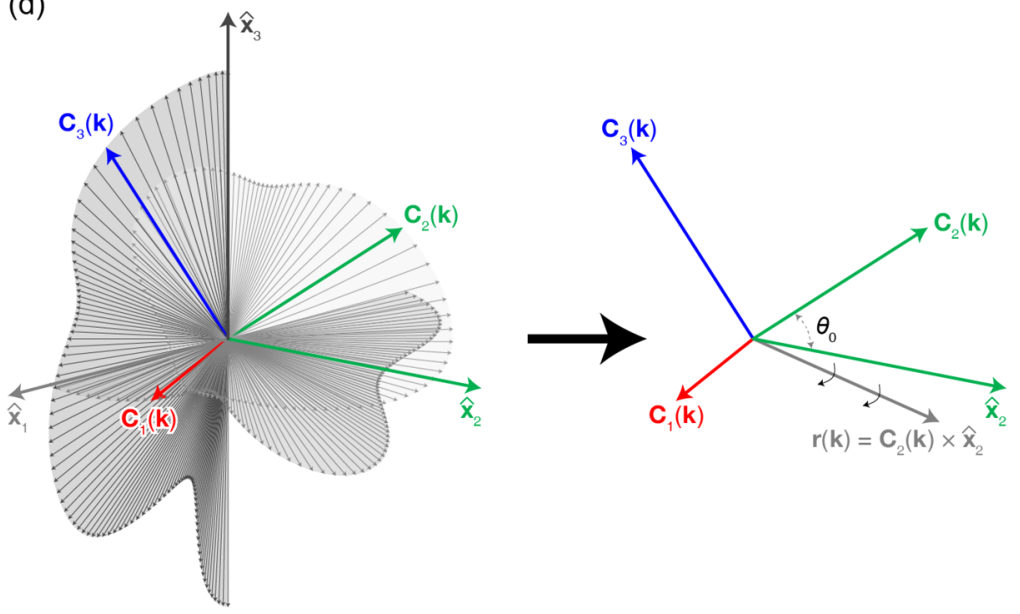

(e)

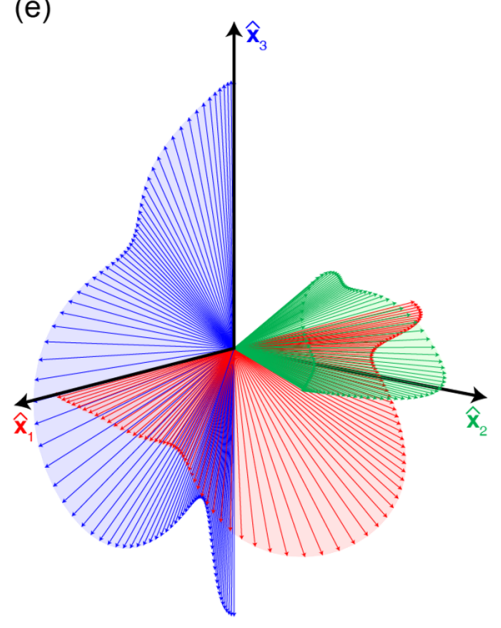

(f)
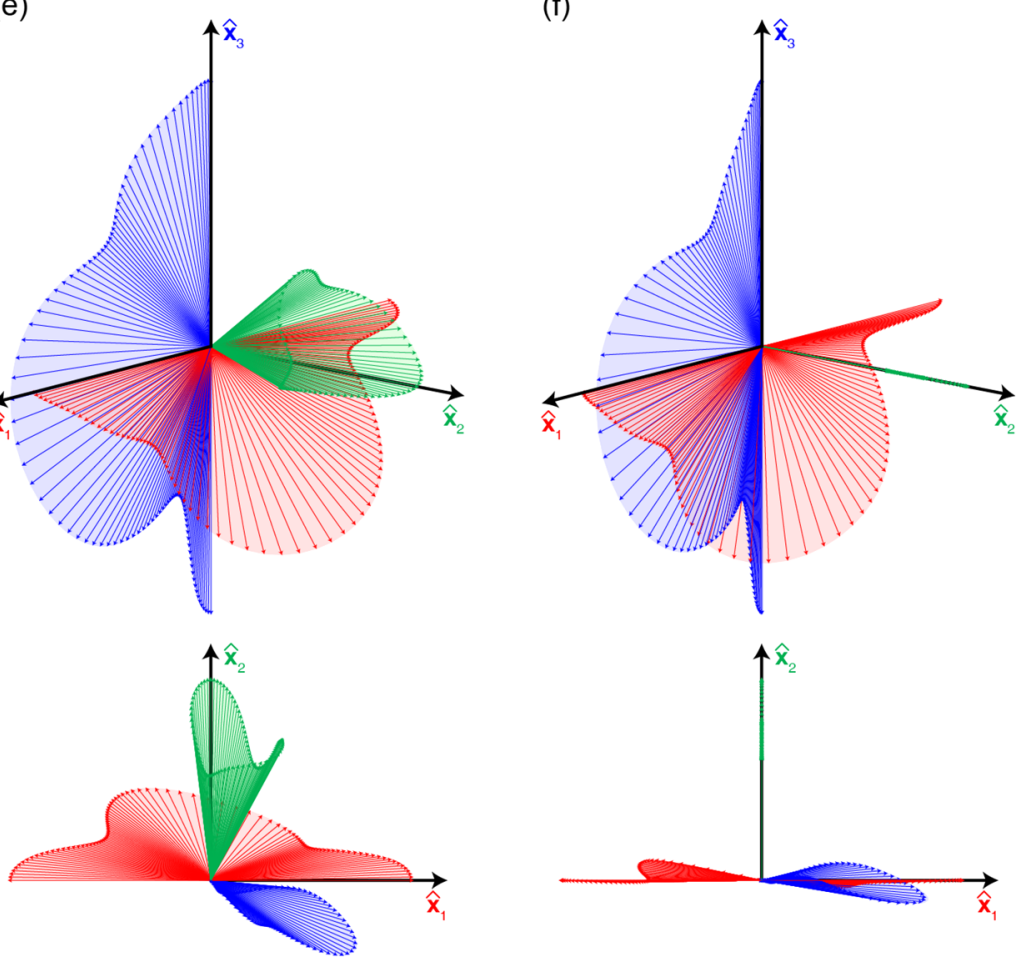

Figure 3. Derivation of the topological charge j. (a) Closed loop that encloses the cyan and orange nodal rings. Its winding direction and starting point are also marked as an arrow and a circle, respectively. $(\mathrm{b}, \mathrm{c})$ Correlations $\mathbf{C}_{n}$ plotted by the same method in Figure $2 \mathrm{~b}, \mathrm{c}$ and e,f. $(\mathrm{d}) \mathbf{C}_{n}(\mathbf{k})$ at an arbitrary $\mathbf{k}$ and $\hat{\mathbf{x}}_{2}$-axis. The matrix $\mathbf{R}_{\mathrm{C}_{2} \rightarrow \hat{\mathbf{x}}_{2}}(\mathbf{k}, \theta)$ is defined such that this matches $\mathbf{C}_{n}(\mathbf{k})$ onto the $\hat{\mathbf{x}}_{2}$-axis with respect to $\mathbf{r}(\mathbf{k})$ perpendicular to both $\mathbf{C}_{n}(\mathbf{k})$ and $\hat{\mathbf{x}}_{2}$. (e, f) Calibration results of (c) by $\mathbf{R}_{\mathrm{C}_{2} \rightarrow \hat{\mathbf{x}}_{2}}\left(\mathbf{k}, 0.6 \theta_{0}\right)$ and $\mathbf{R}_{\mathrm{C}_{2} \rightarrow \hat{\mathbf{x}}_{2}}\left(\mathbf{k}, \theta_{0}\right)$, respectively, where $\theta_{0}$ is the angle between $\mathbf{C}_{n}(\mathbf{k})$ and $\hat{\mathbf{x}}_{2}$.

the zone boundaries via the $\Gamma$-point. The paths are way off the high symmetry directions of the FCC cell. The band structures along the high symmetry lines commonly show few degeneracy points when they pass near the nodal link (see section 2.1, Supporting Information). This contrasts to the case of the photonic crystal with $A_{1}=A_{2}=A_{3}=1$ and $\gamma=0$ (see section 1.1, Supporting Information); its resulting band structures show high similarity between third and fourth bands, and the degeneracies between fourth and fifth bands always appear around the $\Gamma$-point. For extra degeneracies outside the link shown in Figure 1b, see section 4, Supporting Information. In addition to the nodal link in Figure 1b, the set of degeneracies by the first and second bands forms a nodal chain, as shown in section 6, Supporting Information, and their frequency difference data is in section 7, Supporting Information.

Correlations and Non-Abelian Quaternion Charges k and $\mathbf{i}$. The topological charges of the link can be described by the non-Abelian quaternion group $\mathbb{Q}=\{ \pm 1, \pm \mathbf{i}, \pm \mathbf{j}, \pm \mathbf{k}\}$ .$^{16,42}$ The last three numbers are defined such that $\mathbf{i}^{2}=\mathbf{j}^{2}=$ $\mathbf{k}^{2}=-1$, and their relations are skew-symmetric; $\mathbf{i j}=-\mathbf{j i}=\mathbf{k}, \mathbf{j k}$ $=-\mathbf{k j}=\mathbf{i}$, and $\mathbf{k i}=-\mathbf{i k}=\mathbf{j}$. To observe the topological charges, the third, fourth, and fifth bands are labeled as $n=1,2$, and 3, 


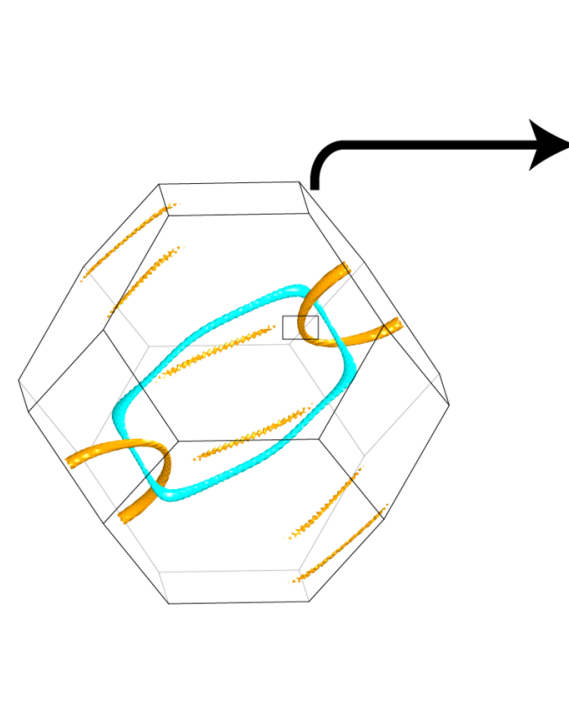

(a)
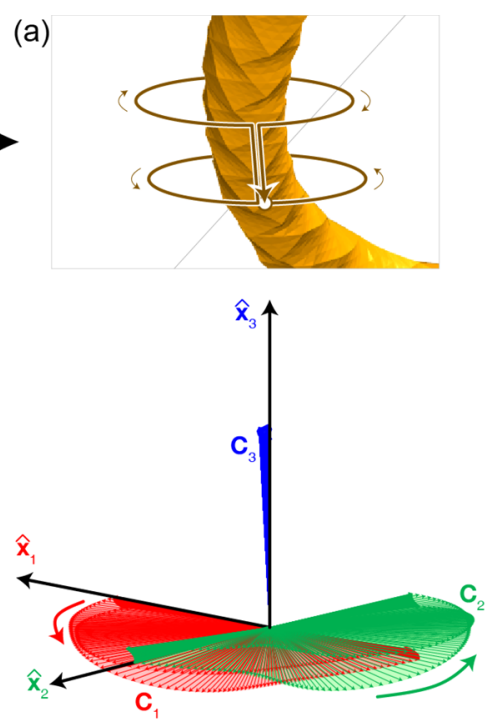

(c)

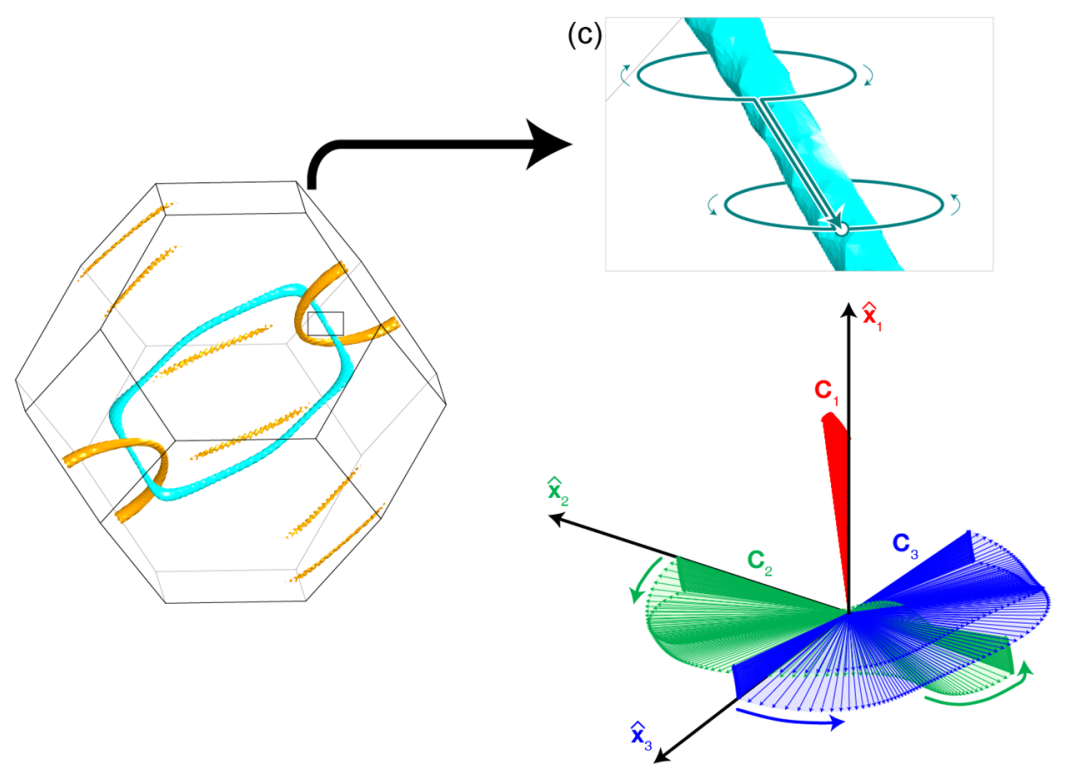

(b)
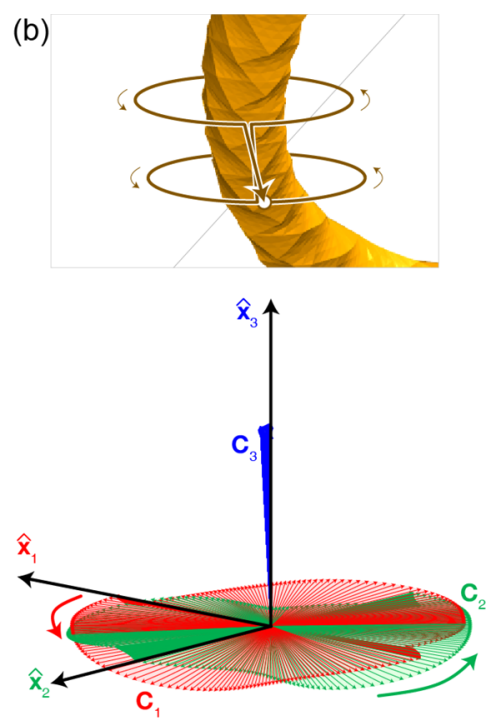

(d)
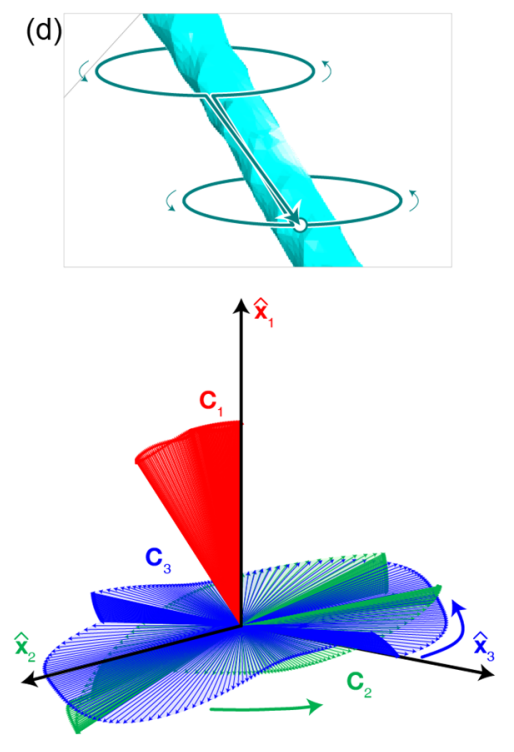

Figure 4. Topological charges with values of \pm 1 . Correlations $\mathbf{C}_{n}$ of the eigenstates along the loops that enclose the orange (a, b) and cyan (c, d) nodes. Only the real parts of the correlations are plotted. All these four panels have two circles in the enlargement insets. The winding directions of these two circles are opposite in (a) and (c) and the same in (b) and (d).

respectively. A closed loop that encircles a point on the orange ring (by the bands $n=1$ and 2) is then considered (Figure 2a). We define the correlation $\mathbf{C}_{n}(\mathbf{k})$ of the band $n$, also known as the polarization, ${ }^{42}$ at any point $\mathbf{k}$ on the closed loop as

$$
\left[\mathbf{C}_{n}(\mathbf{k})\right]_{m}=\left\langle\psi_{\mathbf{k}_{0}}^{m} \mid \psi_{\mathbf{k}}^{n}\right\rangle=\int_{\text {cell }}\left(\psi_{\mathbf{k}_{0}}^{m}\right)^{*} \cdot \psi_{\mathbf{k}}^{n} d^{3} \mathbf{x}
$$

where $\mathbf{k}_{0}$ means the starting point of the closed loop (marked as a circle in Figure $2 \mathrm{a}$ ), and $\psi_{\mathrm{k}}^{n}$ is an eigenstate of the band $n$ such that $\left\langle\psi_{\mathrm{k}}^{m} \mid \psi_{\mathrm{k}}^{n}\right\rangle=\delta_{m n}$. In other words, the correlation $\mathbf{C}_{n}(\mathbf{k})$ $=\left[\left\langle\psi_{\mathbf{k}_{0}}^{1} \mid \psi_{\mathbf{k}}^{n}\right\rangle,\left\langle\psi_{\mathbf{k}_{0}}^{2} \mid \psi_{\mathbf{k}}^{n}\right\rangle,\left\langle\psi_{\mathbf{k}_{0}}^{3} \mid \psi_{\mathbf{k}}^{n}\right\rangle\right]$ of the band $n$ is calculated from the projection of $\left|\psi_{\mathbf{k}}^{n}\right\rangle$ onto $\left|\psi_{\mathbf{k}_{0}}^{m}\right\rangle$. Here, we use $\psi_{\mathbf{k}}^{n}(\mathbf{x})=\mathbf{H}^{n, \mathbf{k}}(\mathbf{x})$, where $\mathbf{H}^{n, \mathbf{k}}(\mathbf{x})$ is the magnetic field eigenstate calculated by MPB. After one winding along the loop, the correlations of the bands $n=1$ and 2 exhibit $\pi$-rotations, while the correlations of the band $n=3$ do not change (Figure $2 \mathrm{~b}$ ). Gathering the tails of all these arrows into the origin generates Figure $2 c$; only $\mathbf{C}_{3}$ remains fixed, while $\mathbf{C}_{1}$ and $\mathbf{C}_{2}$ rotate by $\pi$ around $\mathbf{C}_{3}$. For $\alpha \in$ $[0,2 \pi]$, which parametrizes the closed loop, the rotation matrix for Figure $2 \mathrm{c}$ is expressed as $R_{12}(\alpha)=e^{(\alpha / 2) L_{3}}$, where $\left(L_{i}\right)_{j k}=$ $-\epsilon_{i j k}$. Here, the subscript 12 means that the loop encloses the ring formed by the bands $n=1$ and 2 . Rewriting $R_{12}(\alpha)$ by its lift in the double cover $\operatorname{Spin}(3)$, that is, $\bar{R}_{12}(\alpha)=e^{-i(\alpha / 2)\left(\sigma_{3} / 2\right)}$ with now $\alpha \in[0,4 \pi]$ and substituting $\alpha=2 \pi$ give the topological charge $-i \sigma_{3}$, where $\sigma_{i}$ are the Pauli matrices. ${ }^{16}$ Similar analysis can be carried out on a closed loop which encircles a cyan ring (by the bands $n=2$ and 3 ), as shown in Figure $2 \mathrm{~d}$. In this case, the correlations of the bands $n=2$ and 3 show the $\pi$-rotations, and only the correlations of the band $n$ $=1$ keep the initial direction (Figure 2e,f). Thus, the topological charge $\bar{R}_{23}(2 \pi)$ for Figure $2 \mathrm{f}$ is $-i \sigma_{1}$. 
The topological charges can be calculated using BerryWilczek-Zee connections ${ }^{48}$ instead of calculating the correlations directly. Both methods are equivalent, leading to the same topological charges, as discussed in section 5, Supporting Information.

Non-Abelian Quaternion Charge j. Meanwhile, if we consider a loop that encloses both orange and cyan rings (Figure $3 \mathrm{a}$ ), the $\pi$-rotations occur for the correlations of the bands $n=1$ and 3 (Figure $3 \mathrm{~b}$ ). In contrast to the case of $-i \sigma_{3}$ and $-i \sigma_{1}$ (Figure $2 \mathrm{c}, \mathrm{f}$ ), the correlations in Figure $3 \mathrm{c}$ reveal a more complicated evolution, where $\mathbf{C}_{1}(\mathbf{k})$ and $\mathbf{C}_{3}(\mathbf{k})$ do not rotate on a single plane $\left(\hat{\mathbf{x}}_{1} \hat{\mathbf{x}}_{3}\right.$-plane) and $\mathbf{C}_{2}(\mathbf{k})$ is not fixed along a specific direction ( $\hat{\mathbf{x}}_{2}$-axis). This can be simplified by aligning all $\mathbf{C}_{2}(\mathbf{k})$ along the $\hat{\mathbf{x}}_{2}$-axis as follows. Let us consider $\mathbf{C}_{n}$ at a point $\mathbf{k}$ on the loop (Figure $3 \mathrm{~d}$ ). We can assume a rotation matrix $\mathbf{R}_{\mathbf{C}_{2} \rightarrow \hat{\mathbf{x}}_{2}}(\mathbf{k}, \theta)$, which rotates $\mathbf{C}_{2}(\mathbf{k})$ through an angle $\theta$ with respect to the axis $r(\mathbf{k})=\mathbf{C}_{2}(\mathbf{k}) \times \hat{\mathbf{x}}_{2}$. By applying $\mathbf{R}_{\mathbf{C}_{2} \rightarrow \hat{\mathbf{x}}_{2}}(\mathbf{k}, \theta)$ to not only $\mathbf{C}_{2}(\mathbf{k})$, but also $\mathbf{C}_{1}(\mathbf{k})$ and $\mathbf{C}_{3}(\mathbf{k})$, the correlations in Figure $3 \mathrm{c}$ can be calibrated. If the angle between $\mathbf{C}_{2}(\mathbf{k})$ and $\hat{\mathbf{x}}_{2}$ is denoted as $\theta_{0}(\mathbf{k})$, the correlations transformed by $\mathbf{R}_{\mathrm{C}_{2} \rightarrow \hat{\mathbf{x}}_{2}}\left(\mathbf{k}, 0.6 \theta_{0}\right)$ and $\mathbf{R}_{\mathrm{C}_{2} \rightarrow \hat{\mathbf{x}}_{2}}\left(\mathbf{k}, \theta_{0}\right)$ are shown in Figure $3 \mathrm{e}$ and $\mathrm{f}$, respectively. The topological charge $\bar{R}_{31}(2 \pi)$ for the loop that encloses the two rings is therefore $-i \sigma_{2}$. Because the closed loop first circles around the cyan node $\left(-i \sigma_{2}\right)$ and then around the orange node $\left(-i \sigma_{3}\right)$, the resultant topological charge shown in Figure 3 satisfies the relation $-i \sigma_{2}=$ $\left(-i \sigma_{3}\right)\left(-i \sigma_{1}\right)$. If this sequence is reversed, the sign of this charge may also be flipped. This is discussed in section 3.1, Supporting Information. Meanwhile, the topological charge in Figure 3 can also be drawn without the above calibration, and the discussions are in section 3.2, Supporting Information.

Topological Charges with Values of \pm 1 . The topological charges $I$ and $-I$ can be deduced from the above results. Selecting the same kind of nodes (i.e., same-colored nodes) and setting the closed loop that ties oppositely oriented nodes will generate $\left(-i \sigma_{1}\right)\left(i \sigma_{1}\right)=\left(-i \sigma_{3}\right)\left(i \sigma_{3}\right)=I$. If the loop ties nodes with the same orientation, the result will be $\left(-i \sigma_{1}\right)\left(-i \sigma_{1}\right)=\left(-i \sigma_{3}\right)\left(-i \sigma_{3}\right)=-I$. The former case corresponds to $\alpha$ that increases from zero to $2 \pi$, followed by decreasing to zero. Applying this path to the orange ring makes $\mathbf{C}_{1}$ and $\mathbf{C}_{2}$ rotate by $\pi$ for $\alpha \in[0,2 \pi]$ and by $-\pi$ for $\alpha \in[2 \pi$, $0]$, while keeping $\mathbf{C}_{3}$ (Figure $4 \mathrm{a}$ ). The net evolutions of both $\mathbf{C}_{1}$ and $\mathbf{C}_{2}$ are zero, and therefore the topological charge becomes $I$. The latter case is expressed by setting $\alpha \in[0,4 \pi]$. Along the path, $\mathbf{C}_{1}$ and $\mathbf{C}_{2}$ rotate by $2 \pi$ around $\mathbf{C}_{3}$, as shown in Figure $4 \mathrm{~b}$. This situation is simply written by $\bar{R}_{12}(\alpha)$, as mentioned regarding Figure $2 \mathrm{c}$ in the previous paragraph; substituting $\alpha=4 \pi$ into $\vec{R}_{12}(\alpha)$ gives the topological charge $-I$. The same analyses can be carried out on the cyan ring if only $\mathbf{C}_{1}, \mathbf{C}_{2}$, and $\mathbf{C}_{3}$ in the above sentences are replaced as $\mathbf{C}_{2}, \mathbf{C}_{3}$, and $\mathbf{C}_{1}$, respectively, to generate the results shown in Figure $4 \mathrm{c}, \mathrm{d}$.

All these topological charges can be replaced as quaternions by $-i \sigma_{3} \rightarrow \mathbf{k},-i \sigma_{1} \rightarrow \mathbf{i},-i \sigma_{2} \rightarrow \mathbf{j}, I \rightarrow 1$, and $-I \rightarrow-1$ because the nature of $\left\{ \pm I, \mp i \sigma_{1}, \mp i \sigma_{2}, \mp i \sigma_{3}\right\}$ is isomorphic to $\mathbb{Q}$. Therefore, a closed loop that encloses the ring(s) of the nodal link is classified under the quaternion group.

Surface States. The existence of the nodal link and its non-Abelian charges may open the possibility of observing the topological surface states in our double diamond structure. $^{32,33,40}$ To explore this, the unit cells shown in Figure 1a are stacked along $\mathbf{a}_{2}$ direction. The perfect electric conductor
(PEC) is imposed on both boundaries at the ends of this stacked array. The boundaries are parallel to $\mathbf{a}_{1}$ and $\mathbf{a}_{3}$ directions and these $a_{1}$ and $a_{3}$ are the same directions as $\Gamma \mathrm{K}_{1}$ and $\Gamma \mathrm{K}_{3}$ directions marked in Figure 5a, respectively.

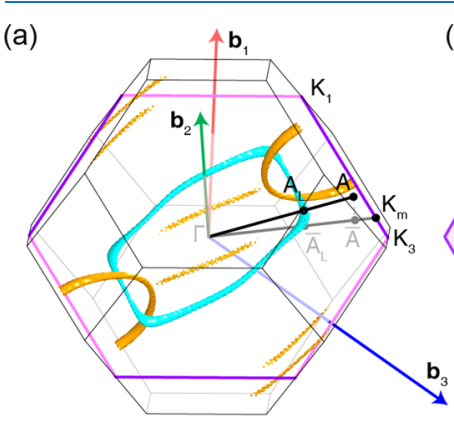

(b)

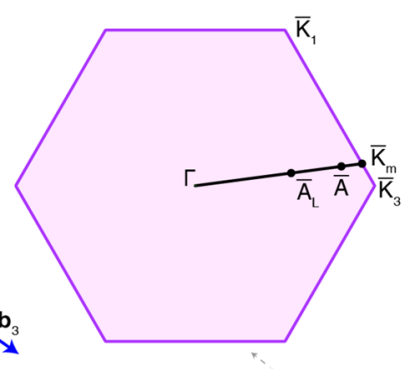

(101) plane

(c)

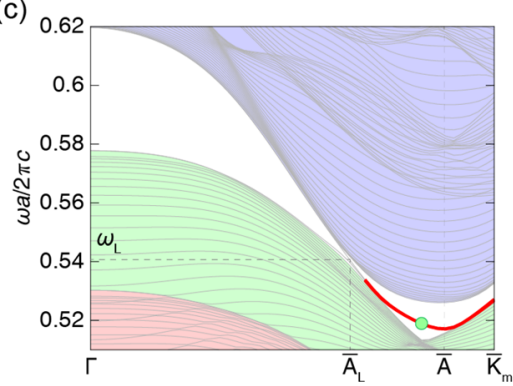

(d)

Figure 5. Surface states on the interface between the double diamond structure and PEC boundary. (a) Bulk Brillouin zone with the nodal link. The magenta-colored hexagon is the (101) surface. The blackcolored path $\Gamma A$ passes the cyan nodal line. Its projection onto the (101) plane is the gray-colored path. (b) Surface Brillouin zone extracted from (a). $\bar{A}_{L}$ and $\bar{A}$ are the projected points of $A_{L}$ and $A$ onto the (101) plane, respectively. (c) Simulated band structure of the 32-layered double diamond structure. The overlapped two bands which correspond surface states are depicted as red. $\omega_{\mathrm{L}}$ is the frequency at $\overline{\mathrm{A}}_{\mathrm{L}}$. (d) The eigenstates $\|\mathbf{H}(\mathbf{x})\|$ at the green point in (c).

Through this bulk-surface correspondence, the nodal link in Figure $5 \mathrm{a}$ is projected to the surface Brillouin zone, shown in Figure $5 \mathrm{~b}$, and the band structure along the $\Gamma \overline{\mathrm{K}}_{\mathrm{m}}$ direction exhibits the projected bulk bands, as shown in Figure 5c (for comparison, see section 2.2, Supporting Information).

In a numerically calculated band structure, Figure 5c, two additional bands, marked in red, appear in the projected bulk bands. These are naturally expected to be surface states, and they are therefore confirmed as the topological surface states because the localizations of the eigenstates $\|\mathbf{H}(\mathbf{x})\|=$ $\sqrt{\mathbf{H}^{*}(\mathbf{x}) \cdot \mathbf{H}(\mathbf{x})}$ around the boundaries are observed as shown in Figure $5 d$, where $\mathbf{H}(\mathbf{x})$ is the magnetic field of the eigenstate.

Although the topological nature of these modes coming from the non-Abelian topological charge is still to be explored, ${ }^{42,47}$ we think that these surface states are related to the quaternion charges through the nodal link. First, the topological charges of the nodal link are expressed by the quaternion numbers, as mentioned throughout this study. Second, the surface states are formed by the nodal link because the surface bands are connected to $\overline{\mathrm{A}}_{\mathrm{L}}$ at $\omega_{\mathrm{L}}$, the frequency and point at which the cyan nodal line passes, respectively. This is 
reminiscent of the edge or surface bands by Dirac or Weyl points, respectively, connected to the frequency and k-points of these points.

\section{CONCLUSIONS}

We have demonstrated the non-Abelian topological nodal link in the momentum space using the double diamond photonic crystal made of dielectric material. The double diamond structure was prepared by breaking geometrical symmetries of the double diamond structure in the space group of $P n \overline{3} m$ (No. 224) except translational and inversion symmetries. We have shown that the reduction of symmetries removes redundant degeneracies that exist in the original double diamond structure. Our double diamond structure still has inversion and time-reversal symmetries, and its conventional unit cell is cubic. We anticipate that different topological properties can be observed by breaking the symmetries or deforming the structure, such as Weyl points by breaking inversion symmetry, ${ }^{12,13}$ time-reversal symmetry, ${ }^{13,57,58}$ or deformation. $^{59}$

The correlation $\mathbf{C}_{n}(\mathbf{k})$ has also been introduced. This quantity acts as a bridge between the non-Abelian band topology and degeneracies of a band structure based on the position-dependent geometry. With the correlation defined from the full vector-field eigenstates and the closed-loop analyses, we have shown that their topological charges are nonAbelian and belong to the quaternion group. Of course, the correlation's usefulness is not limited only to the non-Abelian descriptions of the band theory, but also to the characterization of several phenomena such as surface localizations, ${ }^{31}$ Landau levels, ${ }^{24,60}$ or bound states in the continuum. ${ }^{42}$

Importantly, our results clearly show that the realization of the non-Abelian topological nodal link is not limited by the symmetry and material configurations used in the previous studies on the nodal nodes, including nodal links, ${ }^{33-35,41-45}$ nodal chains, ${ }^{33,34,39-41}$ and nodal knots. ${ }^{35,45,46}$ We believe that our results will lay out the theoretical ground for the experimental observation of photonic non-Abelian charges in purely dielectric materials and allow more freedom in controlling the degeneracy in complex photonic structures.

\section{METHODS}

Simulation of Nodal Link. All photonic band structures and eigenstates in this study, except the data in Figure 5b,c, were obtained by the MPB. ${ }^{56}$ To find the degeneracies, the MPB calculations were performed for the FCC primitive cell in the real space shown in Figure $1 \mathrm{a}$ and a cuboid in the momentum space with $-2 \pi / a \leq k_{x} \leq 2 \pi / a,-2 \pi / a \leq k_{y} \leq 2 \pi /$ $a$, and $0 \leq k_{z} \leq 2 \pi / a$. We used $3 \mathrm{D}$ grids of $16 \times 16 \times 16$ for this primitive cell and $101 \times 101 \times 51$ for this cuboid. The data on the band structure was loaded in MATLAB. We calculated the normalized frequency difference $(\Delta \omega a / 2 \pi c)$ by two adjacent bands and then interpolated the results by using MATLAB's built-in function "interp3". The positive $k_{z}$ region of the nodal link was then plotted by MATLAB's built-in functions "isosurf" and "isocaps". We passed 0.0045 as a tolerance to these two functions via the argument "isovalue"; if a normalized frequency difference of two adjacent bands at $\mathbf{k}$ is smaller than the tolerance, we considered the $\mathbf{k}$ to be degenerated by the bands. The results on this region were copied by inversion symmetry. The plotting was applied only in the first Brillouin zone.
Calculation of Correlations. The MPB calculations on the eigenstates were performed for the closed loops. We used 100 grids (101 points for $\alpha \in[0,2 \pi]$ ) that are uniformly distributed on a closed loop. The magnetic field eigenstates $\mathbf{H}^{n, \mathbf{k}}(\mathbf{x})=\left[H_{1}^{n, \mathbf{k}}(\mathbf{x}), H_{2}^{n, \mathbf{k}}(\mathbf{x}), H_{3}^{n, \mathbf{k}}(\mathbf{x})\right]$ were calculated using the built-in functions "fix-hfield-phase output-hfield" of the MPB. Each output file contains the eigenstates information on the band $n$ at each $\mathbf{k}$. These output files were converted to plain text files using the "h5totxt" utility. For the band $n$ at each $\mathbf{k}$, six plain text files exist that correspond to $\operatorname{Re}\left\{H_{1}^{n, \mathbf{k}}(\mathbf{x})\right\}$, $\operatorname{Im}\left\{H_{1}^{n, \mathbf{k}}(\mathbf{x})\right\}, \operatorname{Re}\left\{H_{2}^{n, \mathbf{k}}(\mathbf{x})\right\}, \quad \operatorname{Im}\left\{H_{2}^{n, \mathbf{k}}(\mathbf{x})\right\}, \operatorname{Re}\left\{H_{3}^{n, \mathbf{k}}(\mathbf{x})\right\}$, and $\operatorname{Im}\left\{H_{3}^{n, \mathbf{k}}(\mathbf{x})\right\}$. Each plain text file consists of $16 \times 16$ lines, and each line has 16 numbers due to the grid size $16 \times 16 \times 16$ mentioned in the previous section. The text files were loaded in MATLAB to produce the correlations by eq 2 . The integral was calculated by the following equation:

$$
\left[\mathbf{C}_{n}(\mathbf{k})\right]_{m}=\int_{\text {cell }}\left(\psi_{\mathbf{k}_{0}}^{m}\right)^{*} \cdot \psi_{\mathbf{k}}^{n} d^{3} \mathbf{x}=\left[\sum_{\mathbf{x}}\left\{H^{m, \mathbf{k}}(\mathbf{x})\right\}^{*} \cdot H^{n, \mathbf{k}}(\mathbf{x})\right] \frac{V}{N^{3}}
$$

where $V=\mathbf{a}_{1} \cdot\left(\mathbf{a}_{2} \times \mathbf{a}_{3}\right)$ is the volume of the FCC primitive cell, and $N^{3}=16 \times 16 \times 16$ is the total number of grids in the cell.

Basically, all eigenstates obtained from an eigenvalue problem exhibit the sign ambiguity, that is, if a state $\left|\psi_{\mathrm{k}}^{n}\right\rangle$ is an eigenstate of a Hamiltonian at $\mathbf{k},-\left|\psi_{\mathbf{k}}^{n}\right\rangle$ is also the eigenstate. Thus, once $\left|\psi_{\mathrm{k}-\Delta \mathrm{k}}^{n}\right\rangle$ was obtained, the signs of $\left|\psi_{\mathrm{k}}^{n}\right\rangle$ for all k-points on the closed loop were determined such that $\operatorname{Re}\left\langle\psi_{\mathbf{k}-\Delta \mathbf{k}}^{n} \mid \psi_{\mathbf{k}}^{n}\right\rangle>0$.

To fix the gauge of $\left|\psi_{\mathrm{k}}^{n}\right\rangle$ on all closed loops, we set a common reference point $\mathbf{k}_{r}=0.5 \Gamma \mathrm{X}+0.15 \Gamma \mathrm{Y}+0.31 \Gamma \mathrm{Z}$, where $\Gamma \mathrm{X}=0.5 \mathbf{b}_{2}+0.5 \mathbf{b}_{3}, \Gamma Y=0.5 \mathbf{b}_{3}+0.5 \mathbf{b}_{1}$, and $\Gamma Z=0.5 \mathbf{b}_{1}$ $+0.5 \mathbf{b}_{2}$. A gauge of $\left|\psi_{\mathbf{k}_{0}}^{n}\right\rangle$ was determined referring to $\left|\psi_{\mathbf{k}_{r}}^{n}\right\rangle$. The MPB calculations mentioned above were also applied here. Detailed discussions on the gauge fixing will be done in a separate study.

Surface States. Figure 5b,c was calculated by COMSOL Multiphysics. We prepared 32 FCC unit cells along the $\mathbf{a}_{2}$ direction. PEC was imposed on two boundaries parallel to both $\mathbf{a}_{1}$ and $\mathbf{a}_{3}$. Other boundaries were set as periodic to the opposite boundaries.

\section{ASSOCIATED CONTENT}

\section{Supporting Information}

The Supporting Information is available free of charge at https://pubs.acs.org/doi/10.1021/acsphotonics.1c00876.

Details for (i) symmetries of the double diamond photonic crystal, (ii) additional band structures, (iii) discussions on the topological charge $\mathbf{j}$, (iv) topological nature on the nodal lines outside the link, (v) topological charges by the Berry-Wilczek-Zee connection, (vi) nodal chain by the double diamond photonic crystal, and (vii) frequency difference data of the nodal chain and nodal link (PDF)

\section{AUTHOR INFORMATION}

\section{Corresponding Author}

Sang Soon Oh - School of Physics and Astronomy, Cardiff University, Cardiff CF24 3AA, United Kingdom; (1) orcid.org/0000-0003-3093-7016; Email: ohs2@ cardiff.ac.uk 


\section{Authors}

Haedong Park - School of Physics and Astronomy, Cardiff University, Cardiff CF24 3AA, United Kingdom; ○ orcid.org/0000-0002-4323-7863

Stephan Wong - School of Physics and Astronomy, Cardiff University, Cardiff CF24 3AA, United Kingdom

Xiao Zhang - School of Physics, Sun Yat-sen University, Guangzhou 510275, China

Complete contact information is available at: https://pubs.acs.org/10.1021/acsphotonics.1c00876

\section{Author Contributions}

S.S.O. and X.Z. conceived this topic; S.S.O. supervised all this work; H.P. proposed the double diamond structure, simulated the photonic band structures, and analyzed the non-Abelian topological charges; S.W. reviewed all mathematical derivations; H.P. and S.S.O. wrote the manuscript; All authors contributed to the discussion of the data and the manuscript.

\section{Notes}

The authors declare no competing financial interest.

\section{ACKNOWLEDGMENTS}

This research was undertaken using the supercomputing facilities at Cardiff University operated by Advanced Research Computing at Cardiff (ARCCA) on behalf of the Cardiff Supercomputing Facility and the HPC Wales and Supercomputing Wales (SCW) projects. We acknowledge the support of the SCW projects and Sêr Cymru II Rising Star Fellowship (80762-CU145 (East)), which are part-funded by the European Regional Development Fund (ERDF) via the Welsh Government. This research was also supported by the National Natural Science Foundation of China (Grant No. 11874431), the National Key R\&D Program of China (Grant No. 2018YFA0306800), and the Guangdong Science and Technology Innovation Youth Talent Program (Grant No. 2016TQ03X688).

\section{REFERENCES}

(1) Slobozhanyuk, A.; Mousavi, S. H.; Ni, X.; Smirnova, D.; Kivshar, Y. S.; Khanikaev, A. B. Three-dimensional all-dielectric photonic topological insulator. Nat. Photonics 2017, 11 (2), 130-136.

(2) Lu, L.; Fang, C.; Fu, L.; Johnson, S. G.; Joannopoulos, J. D.; Soljačić, M. Symmetry-protected topological photonic crystal in three dimensions. Nat. Phys. 2016, 12 (4), 337-340.

(3) Jin, D.; Christensen, T.; Soljačić, M.; Fang, N. X.; Lu, L.; Zhang, X. Infrared Topological Plasmons in Graphene. Phys. Rev. Lett. 2017, 118 (24), 245301.

(4) Liu, G.-G.; Zhou, P.; Yang, Y.; Xue, H.; Ren, X.; Lin, X.; Sun, H.X.; Bi, L.; Chong, Y.; Zhang, B. Observation of an unpaired photonic Dirac point. Nat. Commun. 2020, 11 (1), 1873.

(5) Lu, L.; Wang, Z.; Ye, D.; Ran, L.; Fu, L.; Joannopoulos, J. D.; Soljačić, M. Experimental observation of Weyl points. Science 2015, 349 (6248), 622-624.

(6) Yang, B.; Guo, Q.; Tremain, B.; Liu, R.; Barr, L. E.; Yan, Q.; Gao, W.; Liu, H.; Xiang, Y.; Chen, J.; Fang, C.; Hibbins, A.; Lu, L.; Zhang, S. Ideal Weyl points and helicoid surface states in artificial photonic crystal structures. Science 2018, 359 (6379), 1013-1016.

(7) Yang, Y.; Gao, Z.; Xue, H.; Zhang, L.; He, M.; Yang, Z.; Singh, R.; Chong, Y.; Zhang, B.; Chen, H. Realization of a three-dimensional photonic topological insulator. Nature 2019, 565 (7741), 622-626.

(8) Jia, H.; Zhang, R.; Gao, W.; Guo, Q.; Yang, B.; Hu, J.; Bi, Y.; Xiang, Y.; Liu, C.; Zhang, S. Observation of chiral zero mode in inhomogeneous three-dimensional Weyl metamaterials. Science 2019, 363 (6423), 148-151.
(9) Sie, E. J.; Nyby, C. M.; Pemmaraju, C. D.; Park, S. J.; Shen, X.; Yang, J.; Hoffmann, M. C.; Ofori-Okai, B. K.; Li, R.; Reid, A. H.; Weathersby, S.; Mannebach, E.; Finney, N.; Rhodes, D.; Chenet, D.; Antony, A.; Balicas, L.; Hone, J.; Devereaux, T. P.; Heinz, T. F.; Wang, X.; Lindenberg, A. M. An ultrafast symmetry switch in a Weyl semimetal. Nature 2019, 565 (7737), 61-66.

(10) Soluyanov, A. A.; Gresch, D.; Wang, Z.; Wu, Q.; Troyer, M.; Dai, X.; Bernevig, B. A. Type-II Weyl semimetals. Nature 2015, 527, 495

(11) He, H.; Qiu, C.; Ye, L.; Cai, X.; Fan, X.; Ke, M.; Zhang, F.; Liu, $Z$. Topological negative refraction of surface acoustic waves in a Weyl phononic crystal. Nature 2018, 560 (7716), 61-64.

(12) Park, H.; Lee, S. Double Gyroids for Frequency-Isolated Weyl Points in the Visible Regime and Interference Lithographic Design. ACS Photonics 2020, 7 (6), 1577-1585.

(13) Lu, L.; Fu, L.; Joannopoulos, J. D.; Soljačíc, M. Weyl points and line nodes in gyroid photonic crystals. Nat. Photonics 2013, 7, 294.

(14) Bouhon, A.; Wu, Q.; Slager, R.-J.; Weng, H.; Yazyev, O. V.; Bzdušek, T. Non-Abelian reciprocal braiding of Weyl points and its manifestation in ZrTe. Nat. Phys. 2020, 16 (11), 1137-1143.

(15) Ahn, J.; Kim, D.; Kim, Y.; Yang, B.-J. Band Topology and Linking Structure of Nodal Line Semimetals with $\mathrm{Z}_{2}$ Monopole Charges. Phys. Rev. Lett. 2018, 121 (10), 106403.

(16) Wu, Q.; Soluyanov, A. A.; Bzdušek, T. Non-Abelian band topology in noninteracting metals. Science 2019, 365 (6459), 12731277.

(17) Xia, L.; Guo, Q.; Yang, B.; Han, J.; Liu, C.-X.; Zhang, W.; Zhang, S. Observation of Hourglass Nodal Lines in Photonics. Phys. Rev. Lett. 2019, 122 (10), 103903.

(18) Tiwari, A.; Bzdušek, T. Non-Abelian topology of nodal-line rings in PT-symmetric systems. Phys. Rev. B: Condens. Matter Mater. Phys. 2020, 101 (19), 195130.

(19) Kim, M.; Jacob, Z.; Rho, J. Recent advances in 2D, 3D and higher-order topological photonics. Light: Sci. Appl. 2020, 9 (1), 130.

(20) Lenggenhager, P. M.; Liu, X.; Tsirkin, S. S.; Neupert, T.; Bzdušek, T. From triple-point materials to multiband nodal links. Phys. Rev. B: Condens. Matter Mater. Phys. 2021, 103 (12), L121101.

(21) Li, F.; Huang, X.; Lu, J.; Ma, J.; Liu, Z. Weyl points and Fermi arcs in a chiral phononic crystal. Nat. Phys. 2018, 14, 30.

(22) Gong, Y.; Wong, S.; Bennett, A. J.; Huffaker, D. L.; Oh, S. S. Topological Insulator Laser Using Valley-Hall Photonic Crystals. ACS Photonics 2020, 7 (8), 2089-2097.

(23) Wong, S.; Saba, M.; Hess, O.; Oh, S. S. Gapless unidirectional photonic transport using all-dielectric kagome lattices. Phys. Rev. Res. 2020, 2 (1), 012011.

(24) Wen, X.; Qiu, C.; Qi, Y.; Ye, L.; Ke, M.; Zhang, F.; Liu, Z. Acoustic Landau quantization and quantum-Hall-like edge states. Nat. Phys. 2019, 15 (4), 352-356.

(25) Peri, V.; Serra-Garcia, M.; Ilan, R.; Huber, S. D. Axial-fieldinduced chiral channels in an acoustic Weyl system. Nat. Phys. 2019, 15 (4), 357-361.

(26) Zhang, X.; Chen, Y.; Wang, Y.; Liu, Y.; Lin, J. Y.; Hu, N. C.; Guan, B.; Lee, C. H. Entangled four-dimensional multicomponent topological states from photonic crystal defects. Phys. Rev. B: Condens. Matter Mater. Phys. 2019, 100 (4), 041110.

(27) Noh, J.; Huang, S.; Leykam, D.; Chong, Y. D.; Chen, K. P.; Rechtsman, M. C. Experimental observation of optical Weyl points and Fermi arc-like surface states. Nat. Phys. 2017, 13 (6), 611-617.

(28) Guo, Q.; You, O.; Yang, B.; Sellman, J. B.; Blythe, E.; Liu, H.; Xiang, Y.; Li, J.; Fan, D.; Chen, J.; Chan, C. T.; Zhang, S. Observation of Three-Dimensional Photonic Dirac Points and Spin-Polarized Surface Arcs. Phys. Rev. Lett. 2019, 122 (20), 203903.

(29) Yang, Y.; Xia, J.-p.; Sun, H.-x.; Ge, Y.; Jia, D.; Yuan, S.-q.; Yang, S. A.; Chong, Y.; Zhang, B. Observation of a topological nodal surface and its surface-state arcs in an artificial acoustic crystal. Nat. Commun. 2019, 10 (1), 5185.

(30) He, H.; Qiu, C.; Cai, X.; Xiao, M.; Ke, M.; Zhang, F.; Liu, Z. Observation of quadratic Weyl points and double-helicoid arcs. Nat. Commun. 2020, 11 (1), 1820. 
(31) Deng, W.; Lu, J.; Li, F.; Huang, X.; Yan, M.; Ma, J.; Liu, Z. Nodal rings and drumhead surface states in phononic crystals. Nat. Commun. 2019, 10 (1), 1769.

(32) Gao, W.; Yang, B.; Tremain, B.; Liu, H.; Guo, Q.; Xia, L.; Hibbins, A. P.; Zhang, S. Experimental observation of photonic nodal line degeneracies in metacrystals. Nat. Commun. 2018, 9 (1), 950.

(33) Chang, G.; Xu, S.-Y.; Zhou, X.; Huang, S.-M.; Singh, B.; Wang, B.; Belopolski, I.; Yin, J.; Zhang, S.; Bansil, A.; Lin, H.; Hasan, M. Z. Topological Hopf and Chain Link Semimetal States and Their Application to $\mathrm{Co}_{2} \mathrm{MnGa}$. Phys. Rev. Lett. 2017, 119 (15), 156401.

(34) Belopolski, I.; Manna, K.; Sanchez, D. S.; Chang, G.; Ernst, B.; Yin, J.; Zhang, S. S.; Cochran, T.; Shumiya, N.; Zheng, H.; Singh, B.; Bian, G.; Multer, D.; Litskevich, M.; Zhou, X.; Huang, S.-M.; Wang, B.; Chang, T.-R.; Xu, S.-Y.; Bansil, A.; Felser, C.; Lin, H.; Hasan, M. Z. Discovery of topological Weyl fermion lines and drumhead surface states in a room temperature magnet. Science 2019, 365 (6459), $1278-1281$.

(35) Lee, C. H.; Sutrisno, A.; Hofmann, T.; Helbig, T.; Liu, Y.; Ang, Y. S.; Ang, L. K.; Zhang, X.; Greiter, M.; Thomale, R. Imaging nodal knots in momentum space through topolectrical circuits. Nat. Commun. 2020, 11 (1), 4385.

(36) Hsu, C. W.; Zhen, B.; Stone, A. D.; Joannopoulos, J. D.; Soljačić, M. Bound states in the continuum. Nat. Rev. Mater. 2016, 1 (9), 16048.

(37) Lu, L.; Joannopoulos, J. D.; Soljačić, M. Topological photonics. Nat. Photonics 2014, 8, 821.

(38) Yang, B.; Bi, Y.; Zhang, R.-X.; Zhang, R.-Y.; You, O.; Zhu, Z.; Feng, J.; Sun, H.; Chan, C. T.; Liu, C.-X.; Zhang, S. Momentum space toroidal moment in a photonic metamaterial. Nat. Commun. 2021, 12 (1), 1784.

(39) Bzdušek, T.; Wu, Q.; Rüegg, A.; Sigrist, M.; Soluyanov, A. A. Nodal-chain metals. Nature 2016, 538 (7623), 75-78.

(40) Yan, Q.; Liu, R.; Yan, Z.; Liu, B.; Chen, H.; Wang, Z.; Lu, L. Experimental discovery of nodal chains. Nat. Phys. 2018, 14 (5), 461464.

(41) Yan, Z.; Bi, R.; Shen, H.; Lu, L.; Zhang, S.-C.; Wang, Z. Nodallink semimetals. Phys. Rev. B: Condens. Matter Mater. Phys. 2017, 96 (4), 041103.

(42) Yang, E.; Yang, B.; You, O.; Chan, H.-C.; Mao, P.; Guo, Q.; Ma, S.; Xia, L.; Fan, D.; Xiang, Y.; Zhang, S. Observation of NonAbelian Nodal Links in Photonics. Phys. Rev. Lett. 2020, 125 (3), 033901.

(43) He, P.; Fu, J.-H.; Zhang, D.-W.; Zhu, S.-L. Double exceptional links in a three-dimensional dissipative cold atomic gas. Phys. Rev. A: At., Mol., Opt. Phys. 2020, 102 (2), 023308.

(44) Xie, Y.; Cai, J.; Kim, J.; Chang, P.-Y.; Chen, Y. Hopf-chain networks evolved from triple points. Phys. Rev. B: Condens. Matter Mater. Phys. 2019, 99 (16), 165147.

(45) Yang, Z.; Chiu, C.-K.; Fang, C.; Hu, J. Jones Polynomial and Knot Transitions in Hermitian and non-Hermitian Topological Semimetals. Phys. Rev. Lett. 2020, 124 (18), 186402.

(46) Bi, R.; Yan, Z.; Lu, L.; Wang, Z. Nodal-knot semimetals. Phys. Rev. B: Condens. Matter Mater. Phys. 2017, 96 (20), 201305.

(47) Guo, Q.; Jiang, T.; Zhang, R.-Y.; Zhang, L.; Zhang, Z.-Q.; Yang, B.; Zhang, S.; Chan, C. T. Experimental observation of non-Abelian topological charges and edge states. Nature 2021, 594 (7862), 195200.

(48) Wilczek, F.; Zee, A. Appearance of Gauge Structure in Simple Dynamical Systems. Phys. Rev. Lett. 1984, 52 (24), 2111-2114.

(49) Chiu, C.-K.; Teo, J. C. Y.; Schnyder, A. P.; Ryu, S. Classification of topological quantum matter with symmetries. Rev. Mod. Phys. 2016, 88 (3), 035005

(50) Wang, D.; Yang, B.; Guo, Q.; Zhang, R.-Y.; Xia, L.; Su, X.; Chen, W.-J.; Han, J.; Zhang, S.; Chan, C. T. Intrinsic in-plane nodal chain and generalized quaternion charge protected nodal link in photonics. Light: Sci. Appl. 2021, 10 (1), 83.

(51) Wohlgemuth, M.; Yufa, N.; Hoffman, J.; Thomas, E. L. Triply Periodic Bicontinuous Cubic Microdomain Morphologies by Symmetries. Macromolecules 2001, 34 (17), 6083-6089.
(52) Angelova, A.; Angelov, B.; Mutafchieva, R.; Lesieur, S. Biocompatible Mesoporous and Soft Nanoarchitectures. J. Inorg. Organomet. Polym. Mater. 2015, 25 (2), 214-232.

(53) Barriga, H. M. G.; Tyler, A. I. I.; McCarthy, N. L. C.; Parsons, E. S.; Ces, O.; Law, R. V.; Seddon, J. M.; Brooks, N. J. Temperature and pressure tuneable swollen bicontinuous cubic phases approaching nature's length scales. Soft Matter 2015, 11 (3), 600-607.

(54) La, Y.; Song, J.; Jeong, M. G.; Cho, A.; Jin, S.-M.; Lee, E.; Kim, K. T. Templated synthesis of cubic crystalline single networks having large open-space lattices by polymer cubosomes. Nat. Commun. 2018, 9 (1), 5327.

(55) Sheng, Q.; Li, Q.; Xiang, L.; Huang, T.; Mai, Y.; Han, L. Double diamond structured bicontinuous mesoporous titania templated by a block copolymer for anode material of lithium-ion battery. Nano Res. 2021, 14, 992-997.

(56) Johnson, S. G.; Joannopoulos, J. D. Block-iterative frequencydomain methods for Maxwell's equations in a planewave basis. Opt. Express 2001, 8 (3), 173-190.

(57) Lu, L.; Gao, H.; Wang, Z. Topological one-way fiber of second Chern number. Nat. Commun. 2018, 9 (1), 5384.

(58) Yang, Z.; Xiao, M.; Gao, F.; Lu, L.; Chong, Y.; Zhang, B. Weyl points in a magnetic tetrahedral photonic crystal. Opt. Express 2017, 25 (14), 15772-15777.

(59) Fruchart, M.; Jeon, S.-Y.; Hur, K.; Cheianov, V.; Wiesner, U.; Vitelli, V. Soft self-assembly of Weyl materials for light and sound. Proc. Natl. Acad. Sci. U. S. A. 2018, 115 (16), E3655-E3664.

(60) Rhim, J.-W.; Kim, K.; Yang, B.-J. Quantum distance and anomalous Landau levels of flat bands. Nature 2020, 584 (7819), 5963. 